\title{
Can the outside-in half-tunnel technique reduce femoral tunnel widening in anterior cruciate ligament reconstruction? A CT study
}

\author{
Riccardo Maria Lanzetti ${ }^{1}$ Domenico Lupariello ${ }^{1}$ Angelo De Carli ${ }^{1}$. \\ Edoardo Monaco $^{1} \cdot$ Matteo Guzzini $^{1} \cdot$ Mattia Fabbri $^{1} \cdot$ Antonio Vadalà ${ }^{1}$. \\ Andrea Ferretti ${ }^{1}$
}

Received: 20 February 2017/ Accepted: 30 March 2017

(c) Springer-Verlag France 2017

\begin{abstract}
Background There are different techniques for drilling the femoral tunnel in the anterior cruciate ligament reconstruction (ACLR), but their influence in the bone tunnel enlargement in unknown. The purpose of this study was to compare two different surgical techniques for evaluating femoral tunnel enlargement in ACLR. The hypothesis was that tunnel placement using the outside-in technique leads to less tunnel enlargement compared to the transtibial technique.

Methods Forty-four patients treated for ACLR between March 2013 and March 2014 were prospectively enrolled in this study. According to the surgical technique, subjects were assigned to Group A (Out-in) or Group B (Transtibial). All patients underwent $\mathrm{CT}$ examination in order to evaluate the femoral tunnel enlargement at four different levels. Moreover, all patients were evaluated with the Lachman test and pivot shift test, and the KT1000 arthrometer was used to measure the anterior laxity of the knee. A subjective evaluation was performed using the 2000 International Knee Documentation Committee Subjective Knee score, Lysholm knee score and Tegner activity scale. All patients were assessed after 24 months of follow-up.

Results At the final follow-up, there were statistically significant differences $(p<0.05)$ in femoral tunnel enlargement between the two groups at all four femoral levels in favor of the out-in group. No statistical significant
\end{abstract}

Domenico Lupariello

domenico.lupariello@gmail.com

1 Orthopaedic Unit and "Kirk Kilgour" Sports Injury Centre, S. Andrea Hospital, University of Rome "La Sapienza", Via di Grottarossa 1035, 00189 Rome, RM, Italy differences were found in the objective and subjective clinical outcomes between the two groups $(p>0.05)$. Conclusions In ACLR with a suspension system, the outside-in technique leads to less enlargement of the femoral tunnel lower than the transtibial technique.

Keywords CT imaging - Femoral tunnel enlargement . Anterior cruciate ligament reconstruction · Drilling technique

\section{Introduction}

Bone tunnel enlargement after anterior cruciate ligament (ACL) reconstruction is well described in the literature. The complications associated with tunnel widening include possible delayed graft integration and secondary knee laxity, as well as the additional costs and risks associated with staged revision surgery, if necessary [1,2]. Nevertheless, the cause of tunnel enlargement is unclear; it is thought to be multifactorial, with mechanical and biological factors playing a role $[3,4]$. Among the biomechanical causes, there are the bungee and windshield wiper effects, which arise due to excess motion of the graft in the bone tunnel, or micromotion at the tunnel aperture by the graft with suspensory fixation [5-7]. However, it is controversial as to whether tunnel enlargement affects the clinical outcome [8]. In a recent study, Weber et al. [9] showed that tunnel enlargement is common after ACL reconstruction and that this phenomenon occurs early at the tunnel aperture in the first 6 weeks post-surgery and becomes stable after 24 weeks without clinical impairment. The femoral tunnel can be drilled using a guide through the tibial tunnel, by using the outside-in technique, or via a medial portal. In the last two decades, the most commonly used method worldwide has been the transtibial technique [10]. In 
fact, most studies on tunnel enlargement with suspension devices have investigated the transtibial technique, and little is known of the outside-in technique [11]. To our knowledge, there are few studies in the literature that have analyzed whether the anterior cruciate ligament reconstruction technique can influence the enlargement of the femoral tunnel. The hypothesis of this study was that the drilling technique can influence femoral enlargement; to support this, we compared the results using two different techniques. Our primary aim was to evaluate, on the basis of CT scans, femoral tunnel enlargement in patients who had been operated on for reconstruction of the anterior cruciate ligament and to whom either the outside-in technique or the transtibial technique had been applied at a mean follow-up time of 24 months. The same fixation device was used for all patients. Our secondary aim was to evaluate whether the tunnel widening technique affects clinical outcomes.

\section{Materials and methods}

\section{Participants}

From March 2013 and March 2014, 44 patients met the inclusion criteria and were prospectively enrolled in the study. Exclusion criteria for all attendees were the following: previous knee surgery, multi-ligament injury, and patients with systemic diseases or connective tissue disorders. The inclusion criteria were: patients with isolate ACL rupture, patients with a graft size of $9 \mathrm{~mm}$ who needed a femoral tunnel with a diameter of $9 \mathrm{~mm}$. On the basis of the surgical technique applied, subjects were assigned to either Group A (out-in technique) or Group B (transtibial technique). The same fixation device (TightRope) was used in both groups. All patients agreed to participate in the study and signed an informed consent form in accordance with the Declaration of Helsinki. The protocol for the study was approved by the Local Ethics and Experimental Research Department.

\section{Surgical technique}

In both groups, surgery was performed using an arthroscopic technique through standard arthroscopic portals. Spinal or general anesthesia was administered on the basis of patient preference. We always gave subjects prophylactic antibiotics and anti-thromboembolics before and after the surgery. The patient was placed in the supine position. We placed an ischemic tourniquet at the root of the lower limb in all patients. After an arthroscopic evaluation of the ACL and meniscal and cartilage tears, an incision was performed at the level of the hamstrings (semitendinosus and gracilis). They were harvested and doubled. The choice of the tunnel diameter was made on the basis of the diameter of the graft.

\section{Outside-in technique}

The femoral tunnel was drilled in an anatomical position that was separated from and unrelated to the tibial tunnel position using the Flipcutter Guide Pin (Arthrex, Nalples, FL, USA). This tool works as a guide wire (3.5 $\mathrm{mm}$ in diameter); it was inserted using with the outside-in technique from the external femoral condyle and while under arthroscopic control, it was placed on the ACL footprint area; then, a retrograde drill cut the half-tunnel without affecting the external femoral cortex. In both techniques, we drilled a tunnel that had two diameters. The proximal one had a diameter of $3.5 \mathrm{~mm}$, which allowed the passage of the plate of the TightRope. The distal tunnel was larger, with a diameter of $9 \mathrm{~mm}$ to allow for the placement of the graft.

\section{Transtibial technique}

Before drilling the femoral tunnel, a 3.5-mm hole was made until it reached the cortical bone of the external femoral condyle; then as described above, we made the femoral tunnel at the length necessary to place the graft. The extra-articular landmark of the tibial tunnel was always $1 \mathrm{~cm}$ above the insertion of the pes anserinus and $1.5 \mathrm{~cm}$ medial to the tibial tubercle. The tibial tunnel was drilled using a standard tibial guide (Arthrotek Inc., Warsaw, IN, USA). An impingement rod was used to prevent the femoral roof from imping on the graft. On the coronal plane, the tibial drill guide was inclined to place one guide wire $55^{\circ}$ relative to the medial joint line of the tibia. The intra-articular point of the tibial guide was placed at the center of the native tibial footprint of the ACL. After the insertion of the guide pin, a tibial tunnel was created using a cannulated reamer having the same diameter as the graft. After the tibial tunnel was established, an offset guide was placed through the tibial tunnel. The femoral tunnel was drilled through the tibial tunnel with the knee flexed at $90^{\circ}$. The tunnel was drilled over the guide pin using a $3.5-\mathrm{mm}$ drill. Then, the length of the tunnel was measured, and a femoral tunnel was drilled to place the graft. In both groups, the remaining process of tibial fixation was completed without any modification; tibial fixation was completed with a screw on the tibial side with a diameter that was $1 \mathrm{~mm}$ greater than the tibial tunnel and $30-35 \mathrm{~mm}$ in total length.

\section{Rehabilitation protocol}

The rehabilitation protocol was the same for both groups. Patients were allowed to walk using crutches the first day 
after surgery. All patients used a rehabilitation brace for 4 weeks. Patients began to perform terminal extension and active quadriceps isometric exercises immediately after the operation. Full weight bearing was allowed as soon as tolerated. Patients began to engage in full flexion and gradual ambulation without the use of the brace after the fourth postoperative week. Unrestricted return to physical and daily activity was allowed anywhere between 5 and 7 months after surgery.

\section{Clinical evaluation}

An objective assessment of stability was performed by administering the Lachman test, the pivot shift test and the 2000 IKDC knee examination. With the aim of evaluating the results of ACL reconstruction as objectively as possible, a KT1000 arthrometer was used to measure the laxity of the knee by applying manual maximum and a $30-1 b$ force, and then, side-to-side differences were recorded. The subjective evaluation carried out using the 2000 International Knee Documentation Committee (IKDC) Subjective Knee score, the Lysholm knee score and the Tegner activity scale. All patients were assessed after 24 months of follow-up by an assessor who was not the patient's surgeon.

\section{Radiological evaluation}

All patients underwent CT examination at 24 month-followup, using a Philips computerized tomographer (MX 800016 layers; GE Light Speed 16 layers) in order to study and evaluate the change in the diameter of the femoral tunnel in accordance with the CT protocol drafted by Ferretti et al. $[9,12-14]$. The scan was performed on a section that included the area of the femoral tunnel, adding $2 \mathrm{~cm}$ of additional margin. The slice thickness was $1 \mathrm{~mm}$ with retro reconstruction of $0.65 \mathrm{~mm}$. Measurements were taken at four different levels [9, 12-14]. Images were obtained using a volumetric mode; the volume was scanned, and the raw data sets were subsequently manipulated, thus allowing for postprocess reformation along all the axes (perpendicular, horizontal and oblique). All diameters were calculated in millimeters. A blinded radiologist made all the measurements.
Four scans determined the tunnel diameter: (1) F1: femoral tunnel at the notch, axial image, (2) F2: femoral tunnel at the middle third, axial image, (3) F3: femoral tunnel at the middle point, on the sagittal image reconstruction, (4) F4: femoral tunnel at the middle point, on the coronal image reconstruction. The values of the groups were compared considering, at time 0 (T0), the tunnel measurement of $9 \mathrm{~mm}$ (diameter of the drill used for the half-tunnel in all patients).

\section{Sample size}

An a priori power analysis was performed in accordance with the femoral tunnel enlargement values of CT scans on axial images. Assuming a two-tailed $\alpha$-value of 0.05 (sensitivity $95 \%$ ), a $\beta$ value 0.20 (study power: $80 \%$ ) and an effect size value of 0.70 , we determined that at least 22 patients were required for each group (G Power3 power analysis program).

\section{Statistical analysis}

All the data were analyzed by a single researcher. We used parametric tests when data were normally distributed and homogeneous; we used nonparametric tests when these two conditions were not satisfied. These assumptions were assessed by the Kolmogorov-Smirnov test and Levene test, respectively. An unpaired $t$ test was used to evaluate the differences between the two groups in accordance with the tunnel enlargement values from the $\mathrm{CT}$ scans and with clinical examination results. The Statistical Package for Social Sciences (SPSS) version 22 was used for calculations. Differences with $p$-values $\leq 0.05$ were considered to be statistically significant, and all results are expressed with a $95 \%$ confidence interval.

\section{Results}

The demographic and clinical characteristics of the groups are summarized in Table 1. The groups were homogenous at baseline with regard to age, gender, BMI, dominance and disease duration. Forty-four patients, 22 in Group A [ 16 men and 6 women; mean age (standard deviation, $\mathrm{SD})=26.23(2.34)$;
Table 1 Baseline characteristics of the patients

\begin{tabular}{llll}
\hline & Group A & Group B & $p$ \\
\hline Age & $26.23 \pm 2.34$ & $25.18 \pm 3.55$ & n.s. \\
Gender & & & \\
Male & 16 & 17 & n.s. \\
Female & 6 & 5 & n.s. \\
Dominant limb & 12 & 12 & n.s. \\
BMI $\left(\mathrm{kg} / \mathrm{m}^{2}\right)$ & $22.7 \pm 2.1$ & $23.2 \pm 2.5$ & n.s. \\
Tegner activity level before injury & 7 (range 6.5-8) & 6.5 (range 6-8) & n.s. \\
\hline
\end{tabular}

There were no differences in the baseline features between Group A and Group B $(p>0.05)$ 
Table 2 Clinical evaluation

\begin{tabular}{llll}
\hline & Group A & Group B & $p$ \\
\hline $\begin{array}{l}\text { 2000 IKDC } \\
\text { Subjective }\end{array}$ & $92 \pm 5$ & $90.4 \pm 4$ & n.s. \\
Lysholm & $93.6 \pm 3$ & $93.2 \pm 3$ & n.s. \\
score & (range 82-96) & (range 80-97) & \\
Current & 7 (range 6.5-8) & 6.5 (range 6-8) & n.s. \\
Tegner & Negative & & \\
Lachman test & Negative 20 patients & Negative & n.s. \\
Pivot shift & Positive 2 patients & Positive 1 patient & n.s. \\
& $1.8 \pm 1.1$ & $2.1 \pm 1.2$ & n.s. \\
KT 1000 mm S/S & $2.1 \pm 0.8$ & $2.3 \pm 1$ & n.s. \\
KT 1000 manual & n.s.
\end{tabular}

No significant differences in the clinical evaluations between Group A and Group B ( $p>0.05)$

Table 3 Femoral tunnel enlargement values based on CT scans

\begin{tabular}{lllr}
\hline & Group A & Group B & \multicolumn{1}{l}{$p$} \\
\hline F1 $(\mathrm{mm})$ & $9.16 \pm 0.14$ & $10.56 \pm 0.60$ & 0.022 \\
F2 $(\mathrm{mm})$ & $9.15 \pm 0.15$ & $11.08 \pm 0.66$ & $<0.001$ \\
F3 $(\mathrm{mm})$ & $9.17 \pm 0.09$ & $10.95 \pm 0.83$ & $<0.001$ \\
F4 $(\mathrm{mm})$ & $9.28 \pm 0.13$ & $11.15 \pm 0.66$ & $<0.001$ \\
\hline
\end{tabular}

Significant differences between the two groups at all levels in the CT evaluation of femoral tunnel enlargement

$F 1$ femoral tunnel at the notch, axial image; $F 2$ femoral tunnel at the middle third, axial image; $F 3$ femoral tunnel at the middle point, on the sagittal image reconstruction; $F 4$ femoral tunnel at the middle point, on the coronal image reconstruction

range 18-32] and 22 in Group B [17 men and 5 women mean age $(\mathrm{SD})=25.18(3.55)$; range $18-33$ ], were treated in accordance with the study protocols. No postoperative complications were recorded. Eight selective meniscectomies were performed (six medial meniscus and two lateral meniscus) in Group A, and nine selective meniscectomies were performed (eight medial meniscus and one lateral meniscus) in Group B. The femoral half-tunnel length was $27 \mathrm{~mm} \pm 2.1$ in Group A and $31 \mathrm{~mm} \pm 2.7$ in Group B. As far as the clinical outcome measures, no statistically significant differences were found between the two groups $(p>0.05)$ (Table 2). By the last follow-up, $60 \%$ of patients had returned to their original level of sports activity as provided for by the preoperative Tegner activity scales (Table 2). We found significant differences between the two groups in terms of $\mathrm{F} 1(F=13: 22, p=0.022)$, F2 $(F=34.98, p<0.001)$, F3 $(F=19: 24, p<0.001)$, and $\mathrm{F} 4(F=19.86, p<0.001)$ (Table 3$)$.

\section{Discussion}

The most important finding of this study was that the outside-in technique was associated with less femoral tunnel enlargement in patients when compared to the transtibial technique at the final follow-up. Moreover, we found no other differences between the two groups with respect to clinical outcomes (patient-reported and clinical examination outcomes). Thus, the hypothesis of the study was supported. The TightRope system can be placed using either the outside-in or transtibial technique, so we were able to compare two different surgical techniques since the device was the same in both cases. As far as we know, this is the first work in the literature to compare these different techniques of graft femoral drilling with the same fixation device. Tunnel enlargement after ACL reconstruction has been well studied, but its causes are not yet clear. Several authors have shown that it is more evident in the femoral tunnel with respect to the tibial tunnel $[3,15,16]$. This is why we decided to only analyze the femoral tunnel. Tunnel enlargement is caused by biological and mechanical factors. One of the most reliable biological mechanisms is the release of cytokines after intra-articular reconstruction $[15,17]$. In fact, it is well known that inflammatory mediators such as TNF- $\alpha$ activate osteoclasts. A study published in 2004 [18] demonstrated that patients with femoral tunnel enlargement had a high concentration of intra-articular TNF- $\alpha$, IL-6 and NO. In addition, Rodeo et al. [4] found increased osteoclast activity in the tunnel in an animal model. However, mechanical factors also cause tunnel enlargement after ACL reconstruction. Several studies have addressed movement of the graft in the tunnel as a result of enlargement [19, 20]. Some authors attribute this phenomenon to the rigidity of the fixation system [13, 20], others to the fact that suspension devices facilitate graft movement in the femoral tunnel [19, 21, 22]. The outside-in technique is able to produce shorter tunnels and to reduce the distance of the device from the joint space, thus reducing the windshield wiper effect [20] and the bungee effect [19]. These findings are in agreement with hypotheses proposed by other authors [21, 22], who showed that the greater the distance of the cortical fixation system from the 
joint space, the greater the femoral tunnel enlargement. Another possible explanation for our results is that, during femoral tunnel placement with the conventional transtibial technique, the guide pin and drill often need to be torqued and drilling is not concentric; in the outside-in technique, femoral tunnel placement is not forced by the tibial tunnel, as shown by Chambra et al. [23]. Moreover, in a recent cadaveric study, Osti et al. [24] showed that the outside-in portal surgical technique was superior at positioning the ACL femoral tunnel at the center of the native attachment site when compared with the standard transtibial technique. In fact, drilling the femoral tunnel separately from and unrelated to the tibial tunnel could achieve a more anatomical femoral insertion. This suggests that the outsidein technique may be preferable to the transtibial technique when making a graft corner and furthermore, it could help restore an ACL that is similar to the original one by minimizing graft micromotion, thus resulting in less tunnel widening [19]. This may influence the enlargement of the femoral tunnel, according to our results. In fact, in a recent paper Sim et al. showed that the outside-in technique in ACL reconstruction is recommended over the traditional transtibial technique to prepare the anatomical femoral tunnel in ACL reconstruction [6]. Jagodzinsky et al. [25] have shown how distribution forces are an important factor for femoral tunnel enlargement, assuming these forces are directed and may reduce or increase the enlargement of the tunnel. This might be explained by the fact that, during the normal movement of the knee, the forces and the movement of the graft in the tunnel are more similar to the kinematics of the original articulation. However, further studies are necessary to analyze the correlations between anatomical positioning and tunnel enlargement. Nevertheless, better CT results are not associated with better clinical outcomes; in fact, in our study, both subjective and objective evaluations were quite similar between the two groups. These data are in line with the existing literature. With the exception of Järvelä [5], numerous authors [12-14, 26-30] have shown no correlation between clinical outcomes and the enlargement of the femoral tunnel. Although there are different protocols for the radiological study of enlargement of the femoral tunnel, we chose a protocol using CT since it appears to be the most accurate one as demonstrated by Rathnayaka [14]. The main limitation of our study was the absence of CT immediately after surgery. The choice was determined by the reliability between the cutter diameter and the $\mathrm{CT}$ measurement at $\mathrm{T} 0$, as demonstrated by several authors $[9,13]$. Moreover, this allowed us to reduce radiation exposure. In conclusion, the results of the present study show that, in the reconstruction of the anterior cruciate ligament with a suspension system, the outside-in halftunnel technique results in less enlargement of the femoral tunnel when compared to the transtibial technique. The results obtained from the $\mathrm{CT}$ analysis of the femoral tunnel cannot be correlated with the clinical results, which were excellent in all patients, regardless of the technique used.

\section{Compliance with ethical standards}

Conflict of interest Lanzetti, Lupariello, De Carli, Monaco, Guzzini, Fabbri, Vadalà and Ferretti have nothing to disclose.

Ethical approval All procedures performed in studies involving human participants were in accordance with the ethical standards of the institutional and/or national research committee and with the 1964 Declaration of Helsinki and its later amendments or comparable ethical standards.

Informed consent Informed consent was obtained from all individual participants included in the study.

\section{References}

1. Gifstad T, Foss OA, Engebretsen L, Lind M, Forssblad M, Albrektsen G, Drogset JO (2014) Lower risk of revision with patellar tendon autografts compared with hamstring autografts: a registry study based on 45,998 primary ACL reconstructions in Scandinavia. Am J Sports Med 42(10):2319-2328

2. Brophy RH, Wright RW, Matava MJ (2009) Cost analysis of converting from single-bundle to double-bundle anterior cruciate ligament reconstruction. Am J Sports Med 37(4):683-687

3. Ma CB, Francis K, Towers J, Irrgang J, Fu FH, Harner CH (2004) Hamstring anterior cruciate ligament reconstruction: a comparison of bioabsorbable interference screw and endobutton-post fixation. Arthroscopy 20(2):122-128

4. Rodeo SA, Kawamura S, Kim HJ, Dynybil C, Ying L (2006) Tendon healing in a bone tunnel differs at the tunnel entrance versus the tunnel exit: an effect of graft-tunnel motion? Am J Sports Med 34(11):1790-1800

5. Järvelä T, Moisala AS, Paakkala T, Paakkala A (2008) Tunnel enlargement after double-bundle anterior cruciate ligament reconstruction: a prospective, randomized study. Arthroscopy 24(12):1349-1357

6. Sim JA, Kim JM, Lee S, Bae JY, Seon JK (2015) Comparison of tunnel variability between trans-portal and outside-in techniques in ACL reconstruction. Knee Surg Sports Traumatol Arthrosc. doi:10.1007/s00167-015-3950-8

7. Weber AE, Delos D, Oltean HN et al (2015) Tibial and femoral tunnel changes after ACL reconstruction: a prospective 2-year longitudinal MRI study. Am J Sports Med 43(5):1147-1156

8. Stein DA, Hunt SA, Rosen JE, Sherman OH (2002) The incidence and outcome of patella fractures after anterior cruciate ligament reconstruction. Arthroscopy 18(6):578-583

9. Vadalà A, Iorio R, De Carli A, Argento G, Di Sanzo V, Conteduca F, Ferretti A (2007) The effect of accelerated, brace free, rehabilitation on bone tunnel enlargement after ACL reconstruction using hamstring tendons: a CT study. Knee Surg Sports Traumatol Arthrosc 15(4):365-371

10. Spindler KP, Wright RW (2008) Clinical practice: anterior cruciate ligament tear. N Engl J Med 359(20):2135-2142

11. Gottlob CA, Baker CL Jr, Pellissier JM, Colvin L (1999) Cost effectiveness of anterior cruciate ligament reconstruction in young adults. Clin Orthop Relat Res 367:272-282

12. Iorio R, Vadalà A, Argento G, Di Sanzo V, Ferretti A (2007) Bone tunnel enlargement after ACL reconstruction using autologous hamstring tendons: a CT study. Int Orthop 31(1):49-55 
13. Iorio R, Di Sanzo V, Vadalà A, Conteduca J, Mazza D, Redler A, Bolle G, Conteduca F, Ferretti A (2013) ACL reconstruction with hamstrings: how different technique and fixation devices influence bone tunnel enlargement. Eur Rev Med Pharmacol Sci 17(21):2956-2961

14. Vadalà A, Iorio R, De Carli A, Ferretti M, Paravani D, Caperna L, Iorio C, Gatti A, Ferretti A (2013) Platelet-rich plasma: does it help reduce tunnel widening after ACL reconstruction? Knee Surg Sports Traumatol Arthrosc 21(4):824-829

15. Clatworthy MG, Annear P, Bulow JU, Bartlett RJ (1999) Tunnel widening in anterior cruciate ligament reconstruction: a prospective evaluation of hamstring and patella tendon grafts. Knee Surg Sports Traumatol Arthrosc 7(3):138-145

16. Nebelung W, Becker R, Merkel M, Röpke M (1998) Bone tunnel enlargement after anterior cruciate ligament reconstruction with semitendinosus tendon using Endobutton fixation on the femoral side. Arthroscopy 14(8):810-815

17. Wilson TC, Kantaras A, Atay A, Johnson DL (2004) Tunnel enlargement after anterior cruciate ligament surgery. Am J Sports Med 32(2):543-549

18. Zysk SP, Fraunberger P, Veihelmann A, Dörger M, Kalteis T, Maier M, Pellengahr C, Refior HJ (2004) Tunnel enlargement and changes in synovial fluid cytokine profile following anterior cruciate ligament reconstruction with patellar tendon and hamstring tendon autografts. Knee Surg Sports Traumatol Arthrosc 12(2):98-103

19. Höher J, Livesay GA, Ma CB, Withrow JD, Fu FH, Woo SL (1999) Hamstring graft motion in the femoral bone tunnel when using titanium button/polyester tape fixation. Knee Surg Sports Traumatol Arthrosc 7(4):215-219

20. Tsuda E, Fukuda Y, Loh JC, Debski RE, Fu FH, Woo SL (2002) The effect of soft-tissue graft fixation in anterior cruciate ligament reconstruction on graft-tunnel motion under anterior tibial loading. Arthroscopy 18(9):960-967

21. L'Insalata JC, Klatt B, Fu FH, Harner CD (1997) Tunnel expansion following anterior cruciate ligament reconstruction: a comparison of hamstring and patellar tendon autografts. Knee Surg Sports Traumatol Arthrosc 5(4):234-238
22. Muneta T, Sekiya I, Yagishita K, Ogiuchi T, Yamamoto H, Shinomiya K (1999) Two-bundle reconstruction of the anterior cruciate ligament using semitendinosus tendon with endobuttons: operative technique and preliminary results. Arthroscopy 15(6):618-624

23. Chhabra A, Kline AJ, Nilles KM, Harner CD (2006) Tunnel expansion after anterior cruciate ligament reconstruction with autogenous hamstrings: a comparison of the medial portal and transtibial techniques. Arthroscopy 22(10):1107-1112

24. Osti M, Krawinkel A, Ostermann M, Hoffelner T, Benedetto KP (2015) Femoral and tibial graft tunnel parameters after transtibial, anteromedial portal, and outside-in single-bundle anterior cruciate ligament reconstruction. Am J Sports Med 43(9):2250-2258

25. Jagodzinski M, Foerstemann T, Mall G, Krettek C, Bosch U, Paessler HH (2005) Analysis of forces of ACL reconstructions at the tunnel entrance: is tunnel enlargement a biomechanical problem? J Biomech 38(1):23-31

26. Cameron M, Buchgraber A, Passler H, Vogt M, Thonar E, Fu F, Evans CH (1997) The natural history of the anterior cruciate ligament-deficient knee. Changes in synovial fluid cytokine and keratan sulfate concentrations. Am J Sports Med 25(6):751-754

27. Choi NH, Lee JH, Son KM, Victoroff BN (2010) Tibial tunnel widening after anterior cruciate ligament reconstructions with hamstring tendons using Rigidfix femoral fixation and Intrafix tibial fixation. Knee Surg Sports Traumatol Arthrosc 18(1):92-97

28. Fahey M, Indelicato PA (1994) Bone tunnel enlargement after anterior cruciate ligament replacement. Am J Sports Med 22(3):410-414

29. Hogervorst T, van der Hart CP, Pels Rijcken TH, Taconis WK (2000) Abnormal bone scans of the tibial tunnel 2 years after patella ligament anterior cruciate ligament reconstruction: correlation with tunnel enlargement and tibial graft length. Knee Surg Sports Traumatol Arthrosc 8(6):322-328

30. Yamazaki S, Yasuda K, Tomita F, Tohyama H, Minami A (2005) The effect of transforming growth factor-betal on intraosseous healing of flexor tendon autograft replacement of anterior cruciate ligament in dogs. Arthroscopy 21(9):1034-1041 